\title{
Assessment of quality of life and anxiety in heart failure outpatients
}

Maria Polikandrioti ${ }^{1}$, Georgios Panoutsopoulos ${ }^{2}$, Athanasia Tsami ${ }^{3}$, Georgia Gerogianni ${ }^{1}$, Sofia Saroglou ${ }^{1}$, Efthalia Thomai ${ }^{1}$, Ioannis Leventzonis ${ }^{1}$

\begin{abstract}
${ }^{1}$ Nursing Department, University of West Attica, Athens, Greece
${ }^{2}$ Department of Nursing, Faculty of Human Movement and Quality of Life Sciences, University of Peloponnese, Sparta, Lakonia, Greece

${ }^{3}$ RN, MSc Nurse, General University Laiko Hospital, Athens, Greece
\end{abstract}

Submitted: 6 January 2019

Accepted: 18 March 2019

Arch Med Sci Atheroscler Dis 2019; 4: e38-e46

DOI: https://doi.org/10.5114/amsad.2019.84444

Copyright $\odot 2019$ Termedia \& Banach

\section{Abstract}

Introduction: Heart failure (HF) is a chronic disease associated with increased morbidity and mortality. HF prevalence is expected to expand enormously, largely due to population ageing, rising incidence of HF risk factors and increased survival after cardiovascular events. The aim of the study was to assess levels of quality of life (QOL) and anxiety in HF outpatients and the associated factors as well as to explore the impact of anxiety on QOL.

Material and methods: One hundred HF outpatients were enrolled in the study. Data collection was performed by completion of the Minnesota Living with Heart Failure Questionnaire (MLHFQ), the Self-rating Anxiety Scale (SAS) and a questionnaire including patients' characteristics.

Results: Of the $100 \mathrm{HF}$ outpatients, $64 \%$ were men and $66 \%$ above 70 years old. Regarding QOL, at least $50 \%$ of patients scored above 68 (median) in the total score of MLHFQ and in terms of anxiety, $50 \%$ scored above 46 (median) in the SAS. These values indicate a large impact of HF on QOL and a moderate impact of $\mathrm{HF}$ on anxiety. Furthermore, a statistically significant correlation was observed between QOL and anxiety in HF outpatients (rho $>0.6, p<0.001)$. An increase in anxiety score by one unit implies a statistically significant increase in QOL by 1.22 points $(95 \% \mathrm{Cl}: 0.91-1.52, p<0.001)$, after adjustment for potential confounders.

Conclusions: The present findings emphasize the importance of alleviating the emotional burden of anxiety, thus improving patients' QOL.

Key words: heart failure, quality of life, anxiety.

\author{
Corresponding author: \\ Prof. Maria Polikandrioti MD, \\ $\mathrm{PhD}$ \\ Nursing Department \\ University of \\ West Attica \\ 12242 Athens, Greece \\ Phone: +3316972425054 \\ E-mail: mpolik2006@yahoo. \\ com
}

\section{Introduction}

Heart failure (HF) is a chronic and progressive clinical syndrome that has turned out to be a major health problem worldwide. According to estimates, HF is expected to grow largely due to increased life expectancy of these patients, which is mainly attributed to early and more accurate diagnosis as well as to the improved medical treatment including new pharmacological agents [1-3].

The HF affects more than 26 million people globally [1, 2], while in the UK the number of newly diagnosed individuals increased by $12 \%$ from 2002 to 2014 [4]. The HF patients' profile remains diverse, but they are mostly elderly, male, with multiple comorbidities and of low socio-eco- 
nomic status [5]. This debilitating disease is associated with high mortality which progressively increases with advancing age. In the USA, mortality rates after $\mathrm{HF}$ diagnosis are up to $10 \%$ at 30 days, $20-30 \%$ at 1 year and $45-60 \%$ over 5 years of follow-up. Similarly, in Europe, mortality rates reach $11 \%$ and $41 \%$ at 1 year and 5 years of follow-up, respectively [6]. Likewise, a recent study in Greece (2017) showed an annual mortality rate of 24.3\% for hospitalized HF patients and $7.7 \%$ for outpatients [7]. Strikingly more, HF imposes enormous health care costs which in the USA reached around $\$ 31$ billion ( $£ 22.5$ billion) in 2012 [1]. In Greece, the mean annual economic burden per $\mathrm{HF}$ patient is estimated at $€ 4411 \pm 4764$, of which two thirds are attributed to hospital care. It is noteworthy that the annual HF re-hospitalization rate is $42.9 \%$ for those having a prior hospitalization and $19.2 \%$ for outpatients [7].

Nowadays, there is observed a growing effort to improve quality of life (QOL) among HF patients and alleviate the symptom burden, thus having as the ultimate goal to minimize health care expenditures and decrease hospital re-admissions. Therefore, multidisciplinary outpatient management of $\mathrm{HF}$ is essential to improve clinical outcomes. The $\mathrm{QOL}$ is a wide concept concerning whether a disease limits individuals' ability to fulfill normal roles [8-10]. Several factors can be held responsible for diminished QOL in this vulnerable group such as $\mathrm{HF}$ exacerbations, coexisting symptoms, frequent readmissions, gloomy prognosis, poor self-care, low-socio-economic status, limited family or social support and knowledge deficits about disease management $[8,9]$. According to patients' perspectives, the most important QOL-related issues involve independence in daily living, physical and cognitive impairment, symptom management, psychological status and hospitalizations [11].

Depression is the most widely explored determinant of QOL in HF patients. However, anxiety not only precedes or coexists with depression but also overlaps in symptoms, particularly those of physiological nature [12]. Specifically more in HF, anxiety disorders range between $8 \%$ and $18 \%$ and clinically significant anxiety ranges between $17 \%$ and $28.8 \%$ [12]. Anxiety in HF is associated with adverse clinical outcomes mainly through behavioral and physiologic pathways $[12,13]$. The main sources of anxiety in HF include progressive physical symptoms, complex therapeutic regimen, comorbidities, failure of coping mechanisms, social isolation, frustrations with a complicated healthcare system, financial worries, fear of death, hopelessness and loss of control [14].

Research regarding QOL and anxiety in HF outpatients is limited. Indeed, despite advances in HF management, the association between anxiety and QOL in HF outpatients still remains an obscure issue which needs to be addressed in order to provide holistic care to this sensitive population.

The aim of the present study was to: a) assess levels of anxiety and QOL in HF outpatients, b) explore associated factors with QOL and anxiety, c) explore the correlation between $\mathrm{QOL}$ and anxiety and d) evaluate the impact of anxiety on QOL.

\section{Material and methods}

\section{Study population}

The study sample consisted of 100 HF outpatients (64 men and 36 women). The study was cross-sectional and the sample was a convenience one. The present study enrolled $100 \mathrm{HF}$ patients visiting outpatient clinics in two public hospitals of Athens in Greece from February 2017 to June 2017. In the present study only 100 patients were eligible because they visited outpatient clinics to monitor HF and not due to other co-existing disease or surgery.

Criteria for patients' inclusion in the study were as follows : i) age over 18 years old, ii) diagnosis of $\mathrm{HF}$ as it was assessed by the cardiologist and written in the medical record, iii) ability to write, read and understand Greek language, iv) ability to read and sign the consent form, and v) administration of conservative therapy. The exclusion criteria were as follows: i) HF patients with major psychiatric disorder, ii) HF patients with impaired cognition and eye or hearing problems, iii) HF patients waiting in outpatient clinics to be treated for some other disease (already co-existing or not), and iv) HF patients following additionally some other medical treatment (e.g. cardiac implanted device) apart from conservative therapy. In the present study there was no intervention group since this research was cross sectional and merely recorded levels of anxiety and QOL and the associated factors and the correlation between anxiety and QOL. For this reason, we used the Minnesota Living With Heart Failure Questionnaire, which is the most widespread instrument to measure $\mathrm{QOL}$ in HF.

Data collection lasted approximately $15 \mathrm{~min}$ and took place when patients were waiting for their routine follow-up in the outpatient clinic of two public hospitals in Athens.

\section{Data - variables}

Data collected for each patient included: i) socio-demographic characteristics e.g. gender, age, marital status, educational level, occupation, place of residence, living alone, and ii) clinical characteristics e.g. co-existing diseases, degree of information about the state of their health, years from $\mathrm{HF}$ onset, prior hospitalization due to HF and 
the stage of disease according to the New York Heart Association Classification system (NYHA). This classification (NYHA), which is nowadays the most widely used in the clinical area for HF patients, was proposed in 1928 and since then it has been revised several times, most recently in 1994. Participants were classified according to NYHA as follows [15]:

- NYHA I: Patients had cardiac disease but without resulting limitations of physical activity.

- NYHA II: Patients had cardiac disease resulting in slight limitation of physical activity. They are comfortable at rest.

- NYHA III: Patients had cardiac disease resulting in marked limitation of physical activity. They are comfortable at rest.

- NYHA IV: Patients had cardiac disease resulting in inability to carry on any physical activity without discomfort. Symptoms of cardiac insufficiency or of the anginal syndrome may be present even at rest.

\section{Ethical considerations}

Patients who met the entry criteria were informed by the researcher about the purposes of the study and participated only after they had given written consent. Participation in the study was on a voluntary basis and anonymity was preserved. Furthermore, all participants were in formed of their rights to refuse or to discontinue their participation, according to the ethical standards of the Helsinki Declaration of 1983.

\section{Anxiety assessment}

The Self-rating Anxiety Scale (SAS)-Zung was used to assess anxiety in HF outpatients. The SAS scale consists of 20 questions that assess how respondents felt during the previous week. Respondents have the ability to answer each question on a Likert type scale. In each question scores of 1-4 were attributed. Reversed scores are necessary in five questions. The scores attributed to each question are summed leading to a total score. Higher scores indicate higher levels of anxiety [16].

\section{Quality of life assessment}

The Minnesota Living With Heart Failure scale was used to assess QOL of HF outpatients. This scale was proposed in 1986 by the University of Minnesota. The Minnesota scale consists of 21 questions that evaluate $\mathrm{QOL}$ in the last month (4 weeks). Respondents have the ability to answer each question on a Likert-grade scale (with scores of $0-5$ ). In the Minnesota scale, there are two subgroups of questions evaluating: a) the physical state, and b) the mental state. The scores attributed to the questions are summed up separately for physical state, mental state and all together to a total score, leading to three scores in the range 0-40, 0-25, and 0-105, respectively. Higher scores indicate worse QOL $[17,18]$.

\section{Statistical analysis}

Categorical data are presented in absolute and relative (\%) frequencies. Kruskal-Wallis and Mann-Whitney tests were used to evaluate the association between scales and patients' characteristics. Spearman's rho correlation coefficient was used to evaluate the association between $\mathrm{QOL}$ and anxiety. In addition, multiple linear regression was performed to evaluate the impact of anxiety on QOL after adjustment for potential confounders. Results are presented as $\beta$-coefficients and 95\% confidence intervals $(\mathrm{Cl})$. The observed significance level was set to $5 \%$. All statistical analyses were performed with SPSS version 20.

\section{Results}

\section{Descriptive characteristics}

Of the 100 patients enrolled in the study, men accounted for $64 \%, 66 \%$ were above 70 years old, $56 \%$ were married, $50.5 \%$ had primary school education, $70 \%$ were pensioners, $69.7 \%$ lived in Attica and $19.4 \%$ were living alone. In terms of clinical characteristics, $96.9 \%$ of the sample had a co-existing disease, 9 patients were unaware of their health problems, 30\% had suffered from HF 11-15 years, $79.1 \%$ reported to have a prior hospitalization due to HF and finally, $41.8 \%$ were of stage NYHA IV (Tables I and II).

\section{Levels of anxiety and QOL}

Table III presents the results concerning QOL and anxiety. Regarding QOL, at least $50 \%$ of the patients scored above than 68 in the Minnesota total score while $50 \%$ of patients scored above 33 and 15 for physical and mental state in QOL, respectively. In terms of anxiety, at least $50 \%$ of the patients scored above 46 (median) in the SAS scale. These values indicate a large impact of HF on QOL and moderate impact of HF on anxiety.

\section{Characteristics associated with anxiety and QOL}

Table IV shows the associations between QOL and anxiety with patients' characteristics.

In terms of anxiety, a statistically significant association was observed between anxiety score and gender $(p<0.001)$ and NYHA classification $(p<0.001)$. More specifically, women had higher anxiety (median: 50) than men (median: 43) and patients with NYHA IV had higher anxiety (median 52) than those with NYHA I-III (median: 43). 
Total QOL score was statistically significantly associated with NYHA classification $(p<0.001)$. Patients with NYHA IV had statistically significantly higher scores (median: 76) than those with

Table I. Patients' basic characteristics

\begin{tabular}{|c|c|}
\hline Parameter & $N(\%)$ \\
\hline \multicolumn{2}{|l|}{ Gender: } \\
\hline Male & $64(64.0)$ \\
\hline Female & $36(36.0)$ \\
\hline \multicolumn{2}{|l|}{ Age [years]: } \\
\hline $30-40$ & $4(4.0)$ \\
\hline $41-50$ & $4(4.0)$ \\
\hline $51-60$ & $10(10.0)$ \\
\hline $61-70$ & $16(16.0)$ \\
\hline$>70$ & $66(66.0)$ \\
\hline \multicolumn{2}{|l|}{ Marital status: } \\
\hline Married & $56(56.0)$ \\
\hline Single & $8(8.0)$ \\
\hline Divorced & $6(6.0)$ \\
\hline Widowed & $28(28.0)$ \\
\hline Living together & $2(2.0)$ \\
\hline \multicolumn{2}{|l|}{ Education: } \\
\hline Primary school & $50(50.5)$ \\
\hline Secondary school & $27(27.3)$ \\
\hline University & $19(19.2)$ \\
\hline $\mathrm{MSc} / \mathrm{PhD}$ & $3(3.0)$ \\
\hline \multicolumn{2}{|l|}{ Job: } \\
\hline Unemployed & $5(5.0)$ \\
\hline Private employee & $7(7.0)$ \\
\hline Freelancer & $9(9.0)$ \\
\hline Household & $5(5.0)$ \\
\hline Pensioner & $70(70.0)$ \\
\hline Other & $4(4.0)$ \\
\hline \multicolumn{2}{|l|}{ Residence: } \\
\hline Attica & $69(69.7)$ \\
\hline Capital city & $11(11.1)$ \\
\hline Small town & $4(4.0)$ \\
\hline Village & $15(15.2)$ \\
\hline \multicolumn{2}{|l|}{ Living alone: } \\
\hline Yes & $19(19.4)$ \\
\hline
\end{tabular}

NYHA I-III (median: 61.5), which means they had worse QOL.

Physical state was statistically significantly associated with gender $(p=0.011)$, marital status $(p=0.055)$ and NYHA classification $(p<0.001)$. More specifically, women (median: 35 ) had a worse physical state than men (median: 32). Single/divorced/widowed patients (median: 34.5) also had a worse physical state than married ones (median: 32). In addition, patients with NYHA IV had a worse physical state (median: 36$)$ than patients with lower classification (median: 29).

Table II. Patients' clinical characteristics

\begin{tabular}{|c|c|}
\hline Parameter & $N(\%)$ \\
\hline \multicolumn{2}{|c|}{ Other disease: } \\
\hline No & $3(3.1)$ \\
\hline Yes & $95(96.9)$ \\
\hline \multicolumn{2}{|c|}{ Degree of information of the state of health: } \\
\hline High & $33(33.3)$ \\
\hline Enough & $35(35.4)$ \\
\hline A little & $22(22.2)$ \\
\hline Not at all & $9(9.1)$ \\
\hline \multicolumn{2}{|c|}{ Years having the heart problem: } \\
\hline$<2$ & $6(6.0)$ \\
\hline $2-5$ & $22(22.0)$ \\
\hline $6-10$ & $20(20.0)$ \\
\hline $11-15$ & $30(30.0)$ \\
\hline$>15$ & $22(22.0)$ \\
\hline \multicolumn{2}{|c|}{ Previous hospitalization due to HF: } \\
\hline No & $19(20.9)$ \\
\hline Yes & $72(79.1)$ \\
\hline \multicolumn{2}{|l|}{ NYHA: } \\
\hline I & $2(2.0)$ \\
\hline II & $19(19.4)$ \\
\hline III & $36(36.7)$ \\
\hline IV & $41(41.8)$ \\
\hline
\end{tabular}

Table III. Levels of anxiety and QOL in HF patients

\begin{tabular}{|lc|}
\hline Variable & Median (IQR) \\
\hline $\begin{array}{l}\text { Total Score Minnesota Quality of life } \\
\text { (range: 0-105) }\end{array}$ & $68(58-77)$ \\
\hline Physical State (range: 0-40) & $33(28-36)$ \\
\hline Mental State (range: 0-25) & $15(10-18)$ \\
\hline Anxiety - Zung (range: $20-80)$ & $46(39-54)$ \\
\hline
\end{tabular}




\begin{tabular}{|c|c|c|c|c|c|c|c|c|c|c|c|c|c|c|c|}
\hline 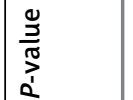 & $\underset{\stackrel{9}{~}}{\overrightarrow{1}}$ & & 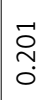 & & $\begin{array}{l}\text { fo } \\
\text { o. }\end{array}$ & & $\begin{array}{l}\stackrel{+}{\stackrel{N}{0}} \\
\stackrel{0}{0}\end{array}$ & & & 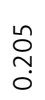 & & & 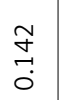 & & \\
\hline 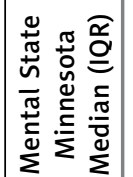 & 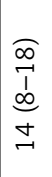 & $\begin{array}{l}\pi \\
n \\
\infty \\
1 \\
1 \\
n \\
= \\
\omega n \\
n \\
n\end{array}$ & 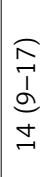 & $\begin{array}{l}\sigma \\
\vec{I} \\
\vec{\Xi} \\
\sim \\
\sim\end{array}$ & 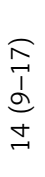 & 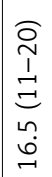 & $\begin{array}{l}\infty \\
0 \\
1 \\
n \\
= \\
0 \\
0 \\
-1\end{array}$ & 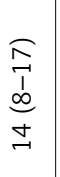 & 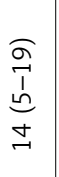 & $\begin{array}{l}\text { F } \\
\vec{j} \\
\vdots \\
0 \\
0 \\
-1\end{array}$ & 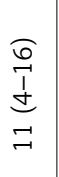 & $\begin{array}{l}\stackrel{\vec{N}}{N} \\
\stackrel{\Xi}{\Xi} \\
\stackrel{n}{\rightarrow}\end{array}$ & $\begin{array}{l}\infty \\
0 \\
\overrightarrow{1} \\
0 \\
\vdots \\
\vec{a}\end{array}$ & 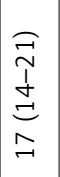 & 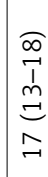 \\
\hline
\end{tabular}

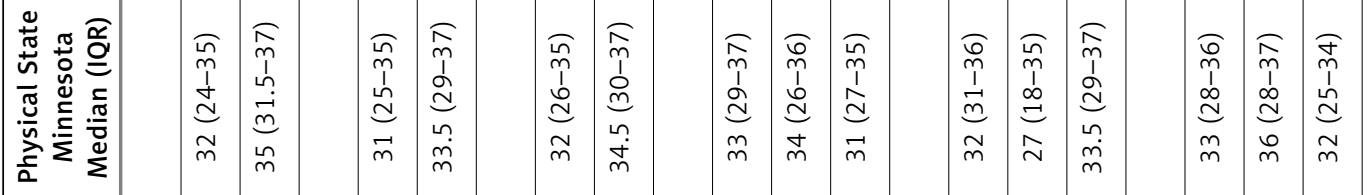

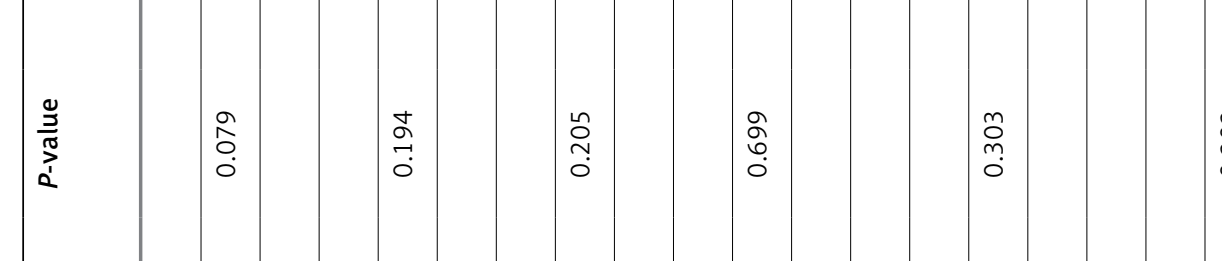

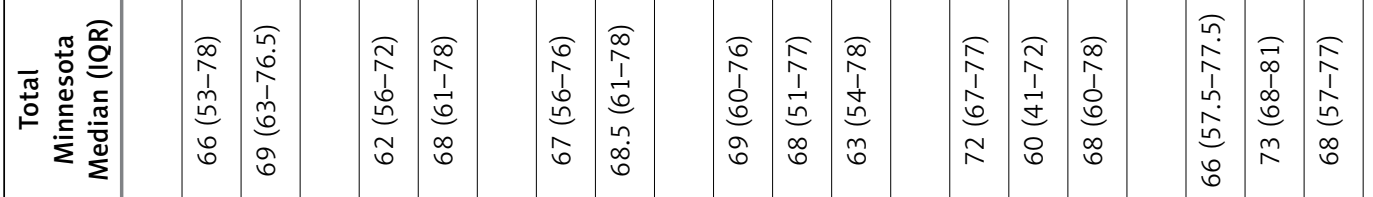

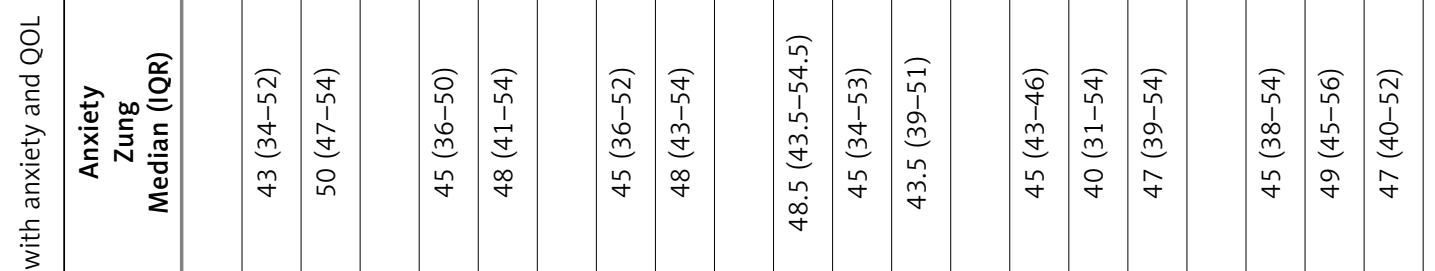

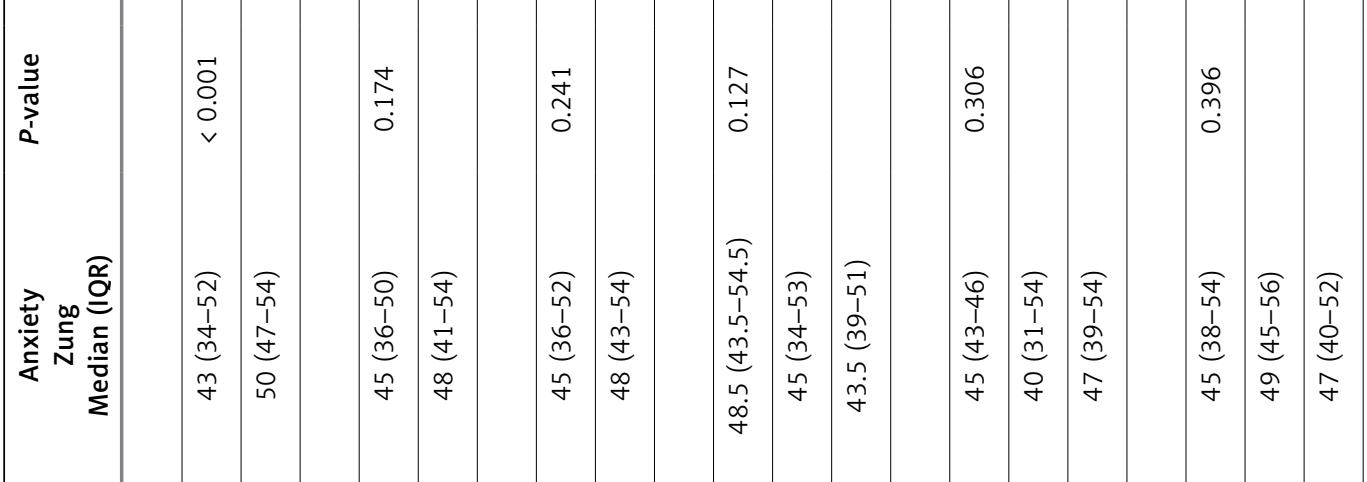

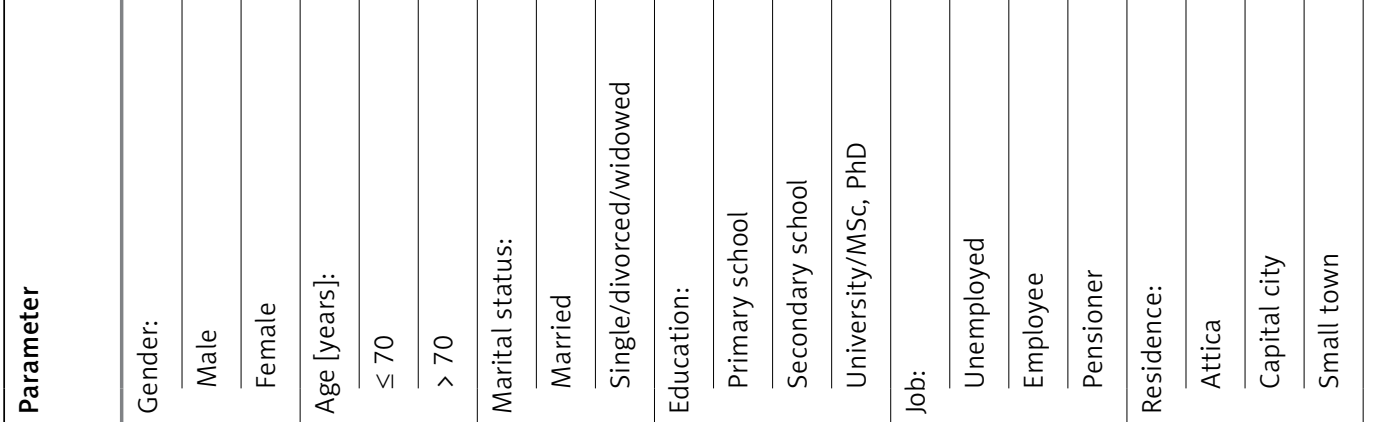




\begin{tabular}{|c|c|c|c|c|c|c|c|c|c|c|c|c|c|c|c|c|c|c|}
\hline 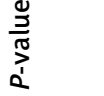 & & 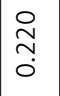 & & & $\stackrel{\stackrel{\infty}{\sim}}{\underset{0}{0}}$ & & & & $\begin{array}{l}\overrightarrow{\vec{b}} \\
\stackrel{0}{0}\end{array}$ & & & & & $\begin{array}{l}\stackrel{n}{\sigma} \\
\stackrel{0}{0}\end{array}$ & & & b. & \\
\hline 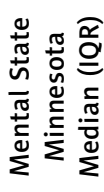 & & $\begin{array}{l}\widehat{E} \\
\overrightarrow{1} \\
\vdots \\
\vdots \\
\vec{J}\end{array}$ & 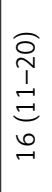 & & $\begin{array}{l}\mathbb{E} \\
\vec{y} \\
\dot{y} \\
m \\
\rightarrow\end{array}$ & 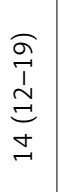 & 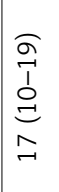 & & 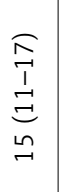 & 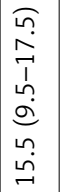 & 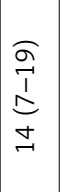 & 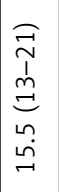 & & 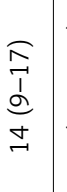 & 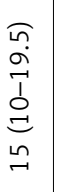 & & & 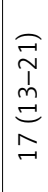 \\
\hline 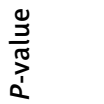 & & 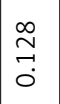 & & & $\begin{array}{l}\vec{\Omega} \\
\hat{0}\end{array}$ & & & & $\begin{array}{l}\overrightarrow{\tilde{n}} \\
\stackrel{0}{0}\end{array}$ & & & & & $\begin{array}{l}\underset{\mathcal{Z}}{0} \\
\stackrel{0}{0}\end{array}$ & & & : & \\
\hline 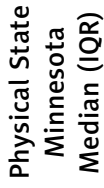 & & 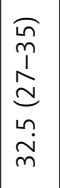 & 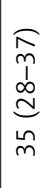 & & 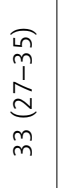 & $\begin{array}{l}\underset{\hat{n}}{m} \\
\stackrel{1}{d} \\
\tilde{n} \\
\tilde{m}\end{array}$ & 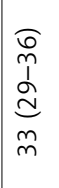 & & 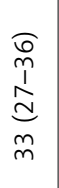 & 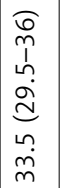 & 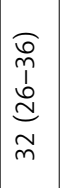 & 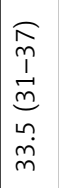 & & 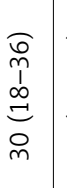 & $\begin{array}{l}\stackrel{0}{\infty} \\
\stackrel{\infty}{\infty} \\
\stackrel{\infty}{\infty} \\
m \\
m\end{array}$ & & & $\begin{array}{l}\infty \\
\infty \\
n \\
1 \\
\tilde{m} \\
e \\
o \\
o \\
m\end{array}$ \\
\hline 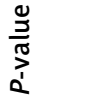 & & 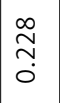 & & & $\begin{array}{l}\vec{\delta} \\
\text { ò }\end{array}$ & & & & $\begin{array}{l}\stackrel{0}{\tilde{e}} \\
\stackrel{\leftrightarrow}{0}\end{array}$ & & & & & $\begin{array}{l}\hat{A} \\
0 \\
0\end{array}$ & & & b. & \\
\hline 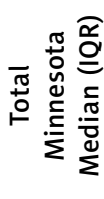 & & 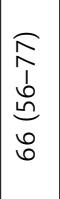 & 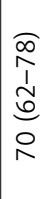 & & 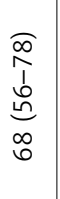 & 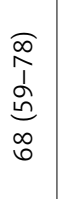 & $\begin{array}{l}0 \\
i \\
1 \\
\hat{n} \\
\hat{0}\end{array}$ & & $\mid \begin{array}{l}\bar{m} \\
\hat{1} \\
0 \\
0 \\
\infty \\
0\end{array}$ & 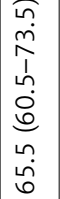 & $\mid \begin{array}{l}\hat{\alpha} \\
\hat{1} \\
0 \\
0 \\
n \\
n \\
\hat{n} \\
0\end{array}$ & 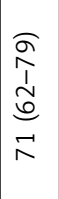 & & 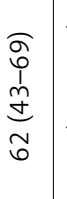 & $\begin{array}{l}\infty \\
\stackrel{\infty}{1} \\
\omega \\
\infty \\
\infty \\
\infty \\
\infty \\
\infty\end{array}$ & & & $\begin{array}{l}\widehat{\sigma} \\
0 \\
1 \\
0 \\
0 \\
0 \\
1 \\
1\end{array}$ \\
\hline $\begin{array}{l}\frac{0}{\tilde{m}} \\
\frac{5}{3} \\
\vdots\end{array}$ & & $\begin{array}{l}\overrightarrow{\tilde{n}} \\
\hat{o} \\
0\end{array}$ & & & 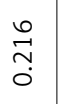 & & & & 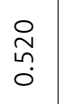 & & & & & 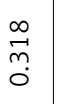 & & & : & \\
\hline 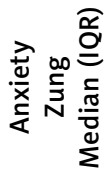 & & $\begin{array}{l}\widehat{N} \\
\hat{i} \\
0 \\
\infty \\
\tilde{m} \\
o \\
o\end{array}$ & 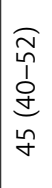 & & 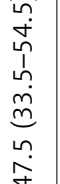 & 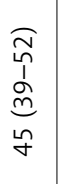 & 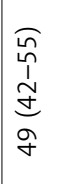 & & 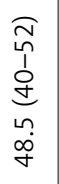 & 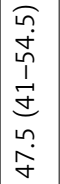 & $\begin{array}{l}\tilde{n} \\
\hat{n} \\
b \\
\tilde{n} \\
m \\
y\end{array}$ & 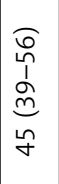 & & 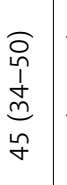 & 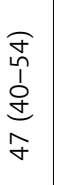 & & & $\begin{array}{l}0 \\
0 \\
n \\
1 \\
n \\
\pm \\
N \\
n\end{array}$ \\
\hline 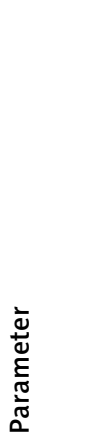 & 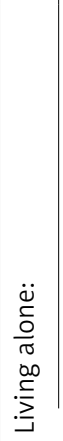 & in & $\stackrel{\breve{\Perp}}{\nu}$ & 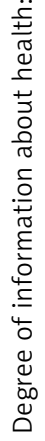 & $\stackrel{\frac{a}{ \pm}}{>}$ & $\begin{array}{l}\frac{\overrightarrow{c o}}{00} \\
\overrightarrow{0} \\
\vec{\omega}\end{array}$ & 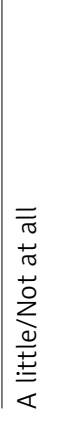 & 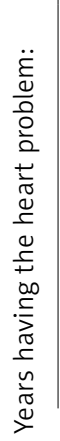 & $\underset{v}{v}$ & 站 & $\stackrel{2}{I}$ & $\stackrel{n}{\stackrel{n}{\wedge}}$ & 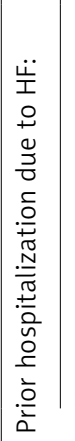 & & $\stackrel{\tilde{\nu}}{>}$ & & & $\geq$ \\
\hline
\end{tabular}


Mental state was statistically significantly associated with marital status $(p=0.047)$ and NYHA classification $(p<0.001)$. The single/divorced/widowed patients (median: 16.5) had a worse mental state than married ones (median: 14). In addition, patients with NYHA IV had a worse mental state (median: 17) than patients with NYHA I-III (median: 13.5).

\section{Association between $\mathrm{QOL}$ and anxiety}

A statistically significant association was found between QOL and anxiety in HF outpatients ( $p<$ 0.001 ) as well as between anxiety and sub-scales of QOL (physical and mental state), $p<0.001$ and $p<0.001$, respectively. The correlation coefficients (rho $>0.6$ ) show a strong positive association. This indicates that the more anxiety patients had, the worse their QOL was (Table V).

\section{Impact of anxiety on QOL}

Multiple linear regression was performed to evaluate the impact of anxiety on QOL of HF patients, adjusting for potential confounders. Factors that were statistically associated univariately with the QOL were introduced as independent variables. Results are presented in Table VI.

We conclude that a one point increase in anxiety score implies a statistically significant increase in QOL score (worsening) by 1.22 points $(95 \% \mathrm{Cl}$ : 0.91-1.52, $p<0.001)$, after adjustment for potential confounders. In addition, patients with NYHA IV have 6.73 points worse QOL than patients with NYHA I-III (95\% Cl: 1.05-12.41, $p=0.021)$.

Similarly, one point increase in anxiety score implies a statistically significant worsening in the physical and mental state by $0.57(95 \% \mathrm{Cl}$ : $0.42-0.72, p<0.001)$ and 0.39 (95\% Cl: 0.27-0.51, $p<0.001)$ points respectively. Finally, patients with NYHA IV have 3.34 points worse physical state than patients with NYHA I-III (95\% Cl: 0.695.98, $p=0.014$ ).

\section{Limitations}

This study has some limitations. Convenience sampling is one of the principal limitations since this method is not representative of all population with HF living in Greece, thus limiting the generalizability of the results. Other limitations are related to the study design, which was cross-sectional and not longitudinal, thus not permitting investigation for a causal relation between anxiety and QOL. Finally, other limitations are: a) the sample size which was relatively small, although many significant associations were observed, b) absence of another measurement that would allow evaluation of possible changes in anxiety and QOL over time, c) comparison with HF hospitalized patients, and d) absence of a control group.

\section{Discussion}

The present results showed large impact of HF on QOL and moderate impact on anxiety. Interestingly, this clinical syndrome is associated with substantial impairment of QOL by reducing patients' ability to undertake prior daily activities or by deteriorating psychosocial state [19-21]. The HF patients define QOL as their ability to perform physical and social activities, to meet their needs, to maintain happiness and fulfill relationships with others [21].

The results also revealed that women had higher anxiety and worse QOL in physical state. QOL and anxiety in HF women represent a growing

Table V. Association between QOL and anxiety

\begin{tabular}{lcc}
\hline Variable & $\begin{array}{c}\text { Anxiety }- \text { Zung } \\
\text { Spearman's rho }\end{array}$ & $P$-value \\
\hline Total Quality of Life & 0.657 & $<0.001$ \\
\hline Physical State & 0.672 & $<0.001$ \\
\hline Mental State & 0.641 & $<0.001$ \\
\hline
\end{tabular}

Table VI. Impact of anxiety on QOL life (adjusted for confounders)

\begin{tabular}{|c|c|c|c|c|c|c|}
\hline Parameter & $\begin{array}{c}\text { Total QOL } \\
\beta \text { coef. }(95 \% \mathrm{Cl})\end{array}$ & $P$-value & $\begin{array}{c}\text { Physical state } \\
\beta \text { coef. }(95 \% \mathrm{Cl})\end{array}$ & $P$-value & $\begin{array}{c}\text { Mental state } \\
\beta \text { coef. }(95 \% \mathrm{Cl})\end{array}$ & $P$-value \\
\hline Anxiety & $1.22(0.91-1.52)$ & $<0.001$ & $0.57(0.42-0.72)$ & $<0.001$ & $0.39(0.27-0.51)$ & $<0.001$ \\
\hline \multicolumn{7}{|l|}{ Gender: } \\
\hline Male & - & & Ref. Cat. & & - & \\
\hline Female & - & & $0.04(-2.7-2.87)$ & 0.977 & - & \\
\hline \multicolumn{7}{|l|}{ Status: } \\
\hline $\begin{array}{l}\text { Single/divorced/ } \\
\text { widowed }\end{array}$ & - & & Ref. Cat. & & Ref. Cat. & \\
\hline Married & - & & $-2.26(-4.8-0.3)$ & 0.084 & $-1.17(-3.2-0.9)$ & 0.264 \\
\hline \multicolumn{7}{|l|}{ NYHA } \\
\hline$|-| I \mid$ & Ref. Cat. & & Ref. Cat. & & Ref. Cat. & \\
\hline IV & $6.73(1.05-12.41)$ & 0.021 & $3.34(0.69-5.98)$ & 0.014 & $1.67(-0.54-3.8)$ & 0.136 \\
\hline
\end{tabular}


area of interest since in the majority of studies, only a small proportion of female patients are enrolled [22-27]. Riegel et al. [27], who explored men and women matched on NYHA classification and age, observed worse QOL in women. This discrepancy may be partially explained by gender differences regarding age, medical history and personal or clinical characteristics. In terms of medical history, women are more likely to have a history of hypertension and a preserved left ventricular function ( $E F>40 \%$ ), have more valvular disease or anemia while compared to other chronic disease, they have more hospital discharges. Significantly more women hardly accept restrictions affecting their ability to support family, thus experiencing more anxiety and diminished QOL. Last but not least, women account for $62 \%$ of total deaths due to HF. By contrast, men are more likely to have a history of coronary artery disease, increased risk factors for atherosclerosis and may develop HF more frequently and at a younger age [22-27].

Single/divorced/widowed patients had worse QOL in physical and mental state when compared to their married counterparts. The mechanism by which marital status affects QOL is believed to be through support and substantial aid to implement behavior change, to promote autonomy, and increase adaptability to disease, thus leading to better clinical outcomes. The HF patients living alone are more vulnerable to psychosocial distress and social isolation, which in turn leads to poor selfcare behaviors [28, 29].

In the present study, the results revealed that NYHA IV was associated with QOL (physical and mental state) and anxiety. Moser et al. [30] found that functional capacity and anxiety predicted poor quality of life in 603 patients in four age groups ( $\leq 53,54-62,63-70$ and $\geq 71$ years). The researchers also observed better QOL among older HF patients, which may be partially attributed to changes in life expectations with advancing age [30].

In terms of anxiety, the results revealed a moderate impact of anxiety on HF patients. Relevant studies in Greece demonstrated $24.7 \%$ and $32.6 \%$ moderate and high levels of anxiety, respectively, in 190 hospitalized HF patients [8] and high levels of both trait and state anxiety in 231 Greek HF outpatients with mean age $66.1 \pm 10.1$ years [13]. Needless to say, anxiety as a normal response to chronic disease has a beneficial effect since it prompts individuals to quickly seek medical help. On the other end of the spectrum, high levels of anxiety are associated with poor prognosis, longer hospital stay, higher risk of coronary events, poor self-care behaviors, non-adherence to dietary and fluid restrictions, undesired pharmacological effects and loss of self confidence [31, 32]. Cardiac patients who confess themselves as anxious may perceive as a threat all the changes or restrictions that impose the disease on their daily living [33]. However, anxiety seems to vary with HF progress. More specifically, at the early stage of HF, patients may experience anxiety due to their inability to continue in prior rhythms, which is increased in advanced stages, due to the fear of death particularly during episodes of dyspnea [19]. Clinical settings should encourage scheduled visits to emphasize cardiac patients' responsibility in the therapeutic regimen.

Data also illustrated that an one point increase in anxiety implies an increase in QOL score (worsening) by 1.22 points. Anxiety may have a direct and an indirect impact on physical and mental state of QOL [34-38]. Elderly HF patients report that disability hinders them from being themselves [37]. Anxiety and type $D$ personality were associated with poor QOL in $459 \mathrm{HF}$ patients (mean age: $66.1 \pm 10.5$ years) [38]. Anxiety independently predicts a decline in physical function over 6 months in $238 \mathrm{HF}$ patients [34] and is also associated with higher re-admission rates, which in turn indicate poor QOL [35]. Moreover, anxiety needs to be addressed since it is associated with alcohol drinking, brain natriuretic peptide, and low social support [36].

In conclusion, the present results showed a large impact of HF on patients' QOL and a moderate impact on patients' anxiety. More specifically, physical state in QOL was associated with gender, marital status, and NYHA classification while mental state was associated with marital status and NYHA classification. Also, the results showed that a one point increase in anxiety score implied an increase in QOL score (worsening) by 1.22 points. A better understanding of the association between anxiety and QOL may help health professionals in planning effective interventions and provide beneficial, holistic care for HF patients.

\section{Conflict of interest}

The authors declare no conflict of interest.

\section{References}

1. Savarese G, Lund LH. Global public health burden of heart failure. Card Fail Rev 2017; 3: 7-11.

2. Cook C, Cole G, Asaria P, et al. The annual global economic burden of heart failure. Int J Cardiol 2014; 171: 368-76.

3. Michalska-Kasiczak M, Bielecka-Dabrowa A, von Haehling S, et al. Biomarkers, myocardial fibrosis and co-morbidities in heart failure with preserved ejection fraction: an overview. Arch Med Sci 2018; 14: 890-909.

4. Wang Q, Dong L, Jian Z, Tang X. Effectiveness of a PRECEDE-based education intervention on quality of life in elderly patients with chronic heart failure. BMC Cardiovasc Disord 2017; 17: 262.

5. Conrad N, Judge A, Tran J, et al. Temporal trends and patterns in heart failure incidence: a population based study of 4 million individuals. Lancet 2018; 391: 572-80. 
6. Bytyçi I, Bajraktari G. Mortality in heart failure patients. Anatol J Cardiol 2014; 15: 63-8.

7. Stafylas P, Farmakis D, Kourlaba G, et al. The heart failure pandemic: the clinical and economic burden in Greece. Int J Cardiol 2017; 227: 923-9.

8. Polikandrioti M, Goudevenos J, Michalis LK, et al. Factors associated with depression and anxiety of hospitalized patients with heart failure. Hellenic J Cardiol 2015; 56: 26-35.

9. Audi G, Korologou A, Koutelekos I, et al. Factors affecting health related quality of life in hospitalized patients with heart failure. Cardiol Res Pract 2017; 2017: 4690458.

10. Carr AJ, Gibson B, Robinson PG. Measuring quality of life: is quality of life determined by expectations or experience? BMJ 2001; 322: 1240-3.

11. Nieminen MS, Dickstein K, Fonseca C, et al. The patient perspective: quality of life in advanced heart failure with frequent hospitalisations. Int J Cardiol 2015; 191: 256-64.

12. Easton K, Coventry P, Lovell K, et al. Prevalence and measurement of anxiety in samples of patients with heart failure: meta-analysis. J Cardiovasc Nurs 20016; 31: 367-79.

13. Aggelopoulou Z, Fotos NV, Chatziefstratiou AA, et al. The level of anxiety, depression and quality of life among patients with heart failure in Greece. ANR 2017; 34: 52-6.

14. De Jong MJ, Chung ML, Wu JR, Riegel B, Rayens MK, Moser DK. Linkages between anxiety and outcomes in heart failure. Heart Lung 2011; 40: 393-404.

15. Raphael C, Briscoe C, Davies J, et al. Limitations of the New York Heart Association functional classification system and self-reported walking distances in chronic heart failure. Heart 2006; 93: 476-82.

16. Samakouri M, Bouhos G, Kadoglou M, et al. Standardization of the Greek version of Zung's Self-Ratting Anxiety Scale. Psychiatriki 2012; 23: 212-20.

17. Brokalaki H, Patelarou E, Giakoumidakis K, et al. Translation and validation of the Greek "Minnesota Living with Heart Failure" questionnaire. Hellenic J Cardiol 2015, 56: 10-9.

18. Rector TS, Kubo SH, Cohn JN. Patients' self-assessment of their congestive heart failure. Part 2: Content, reliability and validity of a new measure, the Minnesota Living with Heart Failure Questionnaire". Heart Failure 1987; 3: 198-209.

19. Jeon $\mathrm{YH}$, Kraus SG, Jowsey $\mathrm{T}$, et al. The experience of living with chronic heart failure: a narrative review of qualitative studies. BMC Health Serv Res 2010; 10: 77.

20. Mendes de Leon CF, Grady KL, Eaton C, et al. Quality of life in a diverse population of heart failure patients: baseline findings from the Heart failure Adherence and Retention Trial (HART). J Cardiopulm Rehabil Prev 2009; 29: 171-8.

21. Heo S, Lennie TA, Okoli C, et al. Quality of life in patients with heart failure: ask the patients. Heart Lung 2009; 38: 100-8.

22. Meyer S, Brouwers FP, Voors AA, et al. Sex differences in new onset heart failure. Clin Res Cardiol 2015; 104: 342-50.

23. Goyal P, Paul T, Almarzooq ZI, et al. Sex and race related differences in characteristics and outcomes of hospitalizations for heart failure with preserved ejection fraction. J Am Heart Assoc 2017; 6: e003330.

24. Riedinger MS, Dracup KA, Brecht ML; SOLVD Investigatos. Studies of left ventricular dysfunction. Quality of life in women with heart failure, normative groups, and patients with other chronic conditions. Am J Crit Care 2002; 11: 211-9.

25. Riedinger MS, Dracup KA, Brecht ML. Quality of life in women with heart failure: do gender differences exist? Heart Lung 2001; 30: 105-16.

26. Stromberg A, Martensson J. Gender differences in patients with heart failure. Eur J Cardiovasc Nurs 2003; 2 7-18.

27. Riegel B, Moser DK, Carlson B, et al. Gender differences in quality of life are minimal in patients with heart failure. J Card Fail 2003; 9: 42-8.

28. Stamp KD, Dunbar SB, Clark PC, et al. Family context influences psychological outcomes of depressive symptoms and emotional quality of life in patients with heart failure. J Cardiovasc Nurs 2014; 29: 517-27.

29. Dunbar SB, Clark PC, Quinn C, et al. Family influences on heart failure self-care and outcomes. J Cardiovasc Nurs 2008; 23: 258-65.

30. Moser DK, Heo S, Lee KS, et al. It could be worse ... lot's worse!' Why health-related quality of life is better in older compared with younger individuals with heart failure. Age Ageing 2013; 42: 626-32.

31. Bentley B, De Jong MJ, Moser DK, et al. Factors related to nonadherence to low sodium diet recommendations in heart failure patients. Eur J Cardiovasc Nurs 2005; 4: 331-6.

32. Brännström M, Ekman I, Norberg A, et al. Living with severe chronic heart failure in palliative advanced home care. Eur J Cardiovasc Nurs 2006; 5: 295-302.

33. Polikandrioti M, Tzirogiannis K, Zyga S, et al. Effect of anxiety and depression on the fatigue of patients with a permanent pacemaker. Arch Med Sci Atheroscler Dis 2018; 3: 8-17.

34. Shen BJ, Eisenberg SA, Maeda U, et al. Depression and anxiety predict decline in physical health functioning in patients with heart failure. Ann Behav Med 2011; 41: 373-82.

35. Tsuchihashi-Makaya M, Kato N, Chishaki A, et al. Anxiety and poor social support are independently associated with adverse outcomes in patients with mild heart failure. Circ J 2009; 73: 280-7.

36. Jokar F, Yousefi H, Yousefy A, et al. Begin again and continue with life: a qualitative study on the experiences of cardiac rehabilitation patients. J Nurs Res 2017; 25 : 344-52.

37. Ekman I, Ehnfors M, Norberg A. The meaning of living with severe chronic heart failure as narrated by elderly people. Scand I Caring Sci 2000; 14: 130-6.

38. Kessing D, Denollet J, Widdershoven J, et al. Self-care and health-related quality of life in chronic heart failure: a longitudinal analysis. Eur J Cardiovasc Nurs 2017; 16: 605-13. 